\title{
Note on upper density of quasi-random hypergraphs
}

\author{
Vindya Bhat \\ Department of Mathematics and Computer Science \\ Emory University \\ Atlanta, U.S.A. \\ vbhat@emory.edu \\ Vojtěch Rödl* \\ Department of Mathematics and Computer Science \\ Emory University \\ Atlanta, U.S.A. \\ rodl@mathcs.emory.edu
}

Submitted: Mar 22, 2013; Accepted: Jun 7, 2013; Published: Jun 21, 2013

Mathematics Subject Classifications: 05C65, 05C42

\begin{abstract}
In 1964, Erdös proved that for any $\alpha>0$, an $l$-uniform hypergraph $G$ with $n \geqslant$ $n_{0}(\alpha, l)$ vertices and $\alpha\left(\begin{array}{l}n \\ l\end{array}\right)$ edges contains a large complete $l$-equipartite subgraph. This implies that any sufficiently large $G$ with density $\alpha>0$ contains a large subgraph with density at least $l ! / l^{l}$.

In this note we study a similar problem for $l$-uniform hypergraphs $Q$ with a weak quasi-random property (i.e. with edges uniformly distributed over the sufficiently large subsets of vertices). We prove that any sufficiently large quasi-random $l$-uniform hypergraph $Q$ with density $\alpha>0$ contains a large subgraph with density at least $\frac{(l-1) !}{l^{l-1}-1}$. In particular, for $l=3$, any sufficiently large such $Q$ contains a large subgraph with density at least $\frac{1}{4}$ which is the best possible lower bound.

We define jumps for quasi-random sequences of $l$-graphs and our result implies that every number between 0 and $\frac{(l-1) !}{l^{l-1}-1}$ is a jump for quasi-random $l$-graphs. For $l=3$ this interval can be improved based on a recent result of Glebov, Král' and Volec. We prove that every number between $[0,0.3192)$ is a jump for quasi-random 3 -graphs.
\end{abstract}

Keywords: hypergraphs; quasi-random; density; jumps Grant

* Research partially supported by NSF grant DMS 1301698 and Emory University Research Committee 


\section{Introduction}

For fixed $l \geqslant 2$, an l-graph $G=(V, E)$ is an $l$-uniform hypergraph with vertex set $V$ and edge set $E \subseteq\left(\begin{array}{c}V \\ l\end{array}\right)$, or a subset of the $l$-tuples of $V$. For $K \subseteq V$ and $|K|=k$, we denote the $l$-subgraph of $G$ induced by $K$ as $G[K]=\left(K, E \cap\left(\begin{array}{c}K \\ l\end{array}\right)\right)$. The density of such an $l$-graph $G$ is defined by $d(G)=|E| /\left(\begin{array}{c}|V| \\ l\end{array}\right)$.

Let $\mathcal{G}=\left\{G_{n}\right\}_{n=1}^{\infty}$ be a sequence of $l$-graphs with $G_{n}=\left(V_{n}, E_{n}\right)$ such that $\left|V_{n}\right| \rightarrow \infty$ as $n \rightarrow \infty$. We define the density $d(\mathcal{G})$ of a sequence $\mathcal{G}$ as $d(\mathcal{G})=\lim _{n \rightarrow \infty} d\left(G_{n}\right)$ (if the limit exists). We will consider only graph sequences for which the limit $d\left(G_{n}\right)$ exists as $n \rightarrow \infty$.

Setting

$$
\sigma_{k}(\mathcal{G})=\max _{n} \max _{K \in\left(\begin{array}{c}
V_{n} \\
k
\end{array}\right)} d\left(G_{n}[K]\right)
$$

a simple averaging argument yields that $\left\{\sigma_{k}(\mathcal{G})\right\}_{k=2}^{\infty}$ is a non-increasing non-negative sequence and so the limit $\bar{d}(\mathcal{G})=\lim _{k \rightarrow \infty} \sigma_{k}(\mathcal{G})$ exists. We call this limit $\bar{d}(\mathcal{G})$ the upper density of $\mathcal{G}$.

The result we present in this note are motivated by a theorem of Erdős from [2]:

Theorem 1.1. For every $\epsilon>0, l \geqslant 2$ and $t$, there exists $n$ such that any l-graph with $n$ vertices and $\epsilon n^{l}$ edges contains a complete l-partite l-graph $K_{t, t, \ldots, t}^{(l)}$. Consequently, for any sequence $\mathcal{G}$ of l-graphs with $d(\mathcal{G})>0, \bar{d}(\mathcal{G}) \geqslant l ! / l^{l}$.

In this note we are interested in a similar problem if we restrict to quasi-random $l$-graphs.

Definition 1.2. Given $\epsilon>0$ and $\alpha>0$, we define an $(\alpha, \epsilon)$-quasi-random hypergraph to be an l-graph $Q=(V, E)$ with the property that for all $W \subseteq V, d(Q[W])=\alpha(1 \pm \epsilon)$ for $|W| \geqslant \epsilon n$ where $|V|=n$. A sequence $\mathcal{Q}=\left\{Q_{n}\right\}_{n=1}^{\infty}$ of $\left(\alpha, \epsilon_{n}\right)$-quasi-random l-graphs is quasi-random if $\epsilon_{n}$ is decreasing and $\epsilon_{n} \rightarrow 0$ as $n \rightarrow \infty$.

Note that for $l=2$ quasi-random graphs must contain arbitrarily large cliques as $\epsilon_{n} \rightarrow 0$ and thus any quasi-random sequence of 2-graphs with $d(\mathcal{Q})>0$ necessarily satisfies $\bar{d}(\mathcal{Q})=1$. In this note we prove a related result for $l \geqslant 3$ :

Theorem 1.3. For a sequence $\mathcal{Q}$ of quasi-random l-graphs with $d(\mathcal{Q})>0$,

(i) $\bar{d}(\mathcal{Q}) \geqslant \frac{(l-1) !}{l^{l-1}-1}$ and

(ii) when $l=3$ there exists a quasi-random sequence of 3-graphs with $\bar{d}(\mathcal{Q})=\frac{1}{4}$.

For $l>3$, however, we do not know if $\bar{d}(\mathcal{Q}) \geqslant \frac{(l-1) !}{l^{l-1}-1}$ could not be replaced by a larger number. Our results for $l=3$ are shown in the Section 2.1 and a similar construction may be applied to generalize the result for all $l$-graphs, proving Theorem 1.3(i).

A number $\alpha$ is a jump if there exists a constant $c=c(\alpha)$ such that given any sequence of l-graphs $\mathcal{G}=\left\{G_{n}\right\}_{n=1}^{\infty}$ if $\bar{d}(\mathcal{G})>\alpha$, then $\bar{d}(\mathcal{G}) \geqslant \alpha+c$. It follows from the Erdös-Stone Theorem that all non-negative numbers less than 1 are jumps for graphs and it follows 
from Theorem 1.1 that all non-negative numbers less than $\frac{l !}{l^{l}}$ are jumps for $l$-graphs. Erdős conjectured that, analogous to graphs, all numbers less than 1 are jumps for $l$-graphs as well. This conjecture was disproved by Frankl and Rödl in [5] who showed that there are an infinite number of non-jumps for all $l \geqslant 3$. However, these non-jumps were found to occur at relatively large densities. While the smallest case of determining whether $\frac{l !}{l^{l}}$ is a jump is still open and likely a difficult problem, our result shows that under the further assumption of quasi-randomness that $\frac{l !}{l^{l}}$ is indeed a jump for all $l \geqslant 3$.

We extend the concept of jumps to sequences of quasi-random $l$-graphs:

Definition 1.4. A number $\alpha$ is a jump for quasi-random l-graphs if there exists a constant $c=c(\alpha)$ such that given any sequence of quasi-random l-graphs $\mathcal{G}=\left\{G_{n}\right\}_{n=1}^{\infty}$ if $\bar{d}(\mathcal{G})>\alpha$, then $\bar{d}(\mathcal{G}) \geqslant \alpha+c$.

Theorem 1.3(i) implies that every number between 0 and $\frac{(l-1) !}{l^{l-1}-1}$ is a jump for quasirandom $l$-graphs. Further we will show that for $l=3$ this interval can be improved from $\left[0, \frac{1}{4}\right)$ to $[0,0.3192)$ given the following question of Erdős [3] is answered positively:

Question 1.5. Let $c>0$ and $\mathcal{Q}=\left\{Q_{n}\right\}_{n=1}^{\infty}$ be a quasi-random sequence of 3-graphs. If $d(\mathcal{Q})=\frac{1}{4}+c$, then does each $Q_{n}$ contain $K_{4}^{(3)}-e$ as $n \rightarrow \infty$ ?

More formally, we prove in Section 3:

Theorem 1.6. A positive answer to Question 1.5 implies that any quasi-random sequence $\mathcal{Q}$ with $d(\mathcal{Q})>\frac{1}{4}$ satisfies $\bar{d}(\mathcal{Q})>0.3192$.

Very recently, Glebov, Král' and Volec in [6] proved Question 1.5 in the positive using Razborov's flag-algebra method [10]. This result confirms our assertion in Theorem 1.6.

We include our remarks and questions for future study for quasi-random $l$-graphs with $l>3$ and other possibilites for jumps for quasi-random 3-graphs in Section 4.

\section{Proof of Theorem 1.3}

\subsection{The lower bound}

Our proof is based on the following lemma proved in [1] and [9]:

Lemma 2.1. For all $\alpha>0$ and $\epsilon>0$, there exists $\delta>0, m>0$ and $n_{0}>0$ such that if $Q=(V, E)$ is an $(\alpha, \delta)$-quasi-random l-graph with $|V|=n \geqslant n_{0}$ vertices then $Q[M]$ is $(\alpha, \epsilon)$-quasi-random for at least $\frac{1}{2}\left(\begin{array}{c}n \\ m\end{array}\right)$ m-sets $M \in\left(\begin{array}{c}n \\ m\end{array}\right)$.

Going forward in this subsection, we restrict to $l=3$ for simplicity. Essentially the same statements may be applied to general l-graphs.

Given a 3-graph $F, \alpha>0$ and $\epsilon>0$, we write $(\alpha, \epsilon) \rightarrow F$ to denote the fact that every $(\alpha, \epsilon)$-quasi-random 3 -graph $R$ contains $F$. Let $F$ and $H$ be 3 -graphs. For $F, H$, and $v \in V(F)$, we define $F_{H}^{v}$ to be the 3-graph as follows: 
(i) $V\left(F_{H}^{v}\right)=V(F) \cup V(H)-v$ and

(ii) $E\left(F_{H}^{v}\right)=E(F-v) \cup E(H) \cup \bigcup_{u \in V(H)}\{\{a, b, u\}:\{a, b, v\} \in E(F)\}$

In other words, to obtain $F_{H}^{v}$ from $F$, replace $v$ with $V(H)$ and add all the edges in $H$ as well as the edges of type $\{a, b, u\}$ where $u \in V(H)$ and $\{a, b, v\} \in E(F)$. In this construction we will assume that $F$ and $H$ are vertex disjoint and thus $\left|V\left(F_{H}^{v}\right)\right|=|V(F)|+|V(H)|-1$ and $\left|E\left(F_{H}^{v}\right)\right|=|E(F)|+|E(H)|+|V(H)-1||\{e \in E(F), v \in e\}|$.

Using the notation stated above, we observe the following:

Lemma 2.2. For all $\alpha>0, \epsilon>0, \gamma>0$ and 3-graphs $F$ and $H$, there exists $\delta=\delta(\alpha, \epsilon, \gamma)>$ 0 such that if $(\alpha, \epsilon) \rightarrow F$ and $(\alpha, \gamma) \rightarrow H$, then $(\alpha, \delta) \rightarrow F_{H}^{v}$.

Proof. Let $|V(F)|=f$ and let $v \in V(F)$. Given $\alpha>0$ and $\epsilon>0$ such that $(\alpha, \epsilon) \rightarrow F$, let $\delta_{L(2.1)}$ and $m=m(\alpha, \epsilon)$ be the constants ensured by Lemma 2.1. Consider an $(\alpha, \delta)$ quasi-random hypergraph $Q$ on $n$ vertices. Set $\delta \leqslant \min \left(\delta_{L(2.1)}, \frac{\gamma}{2 m^{f}}\right)$. We want to show that $Q$ must contain $F_{H}^{v}$. By Lemma 2.1, $R=Q[M]$ is $(\alpha, \epsilon)$-quasi-random for at least $\frac{1}{2}\left(\begin{array}{c}n \\ m\end{array}\right) M$ 's. By assumption $((\alpha, \epsilon) \rightarrow F)$ each such $(\alpha, \epsilon)$-quasi-random $Q[M]$ contains a copy of $F$. Consequently, the number of $Q[M]$ 's with each containing a copy of $F$ is at least $\frac{1}{2}\left(\begin{array}{c}n \\ m\end{array}\right)$. On the other hand, each copy of $F$ is in at most $\left(\begin{array}{c}n-f \\ m-f\end{array}\right)$ different $Q[M]$ 's. Thus, we have at least $\frac{1}{2}\left(\begin{array}{c}n \\ m\end{array}\right) /\left(\begin{array}{c}n-f \\ m-f\end{array}\right)=\frac{\left(\begin{array}{c}n \\ f\end{array}\right)}{2\left(\begin{array}{c}m \\ f\end{array}\right)}>\frac{1}{2}\left(\frac{n}{m}\right)^{f}=c n^{f}$ distinct copies of $F$ in $Q$, where $c=c(m(\alpha, \epsilon), f)=\frac{1}{2 m^{f}}$. Set $V(F)=\left\{u_{1}, u_{2}, \ldots, u_{f-1}, v\right\}$ and let $F^{\text {copy }}=F^{c}$ be a copy of $F$ in $Q$ with $V\left(F^{c}\right)=\left\{u_{1}^{c}, u_{2}^{c}, \ldots, u_{f-1}^{c}, v^{c}\right\}$ so that $u_{i} \rightarrow u_{i}^{c}$ for $i=1,2, \ldots, f-1$ and $v \rightarrow v^{c}$ is an isomorphism.

For each of the $c n^{f}$ copies $F^{c}$ of $F$, consider an ordered $(f-1)$-tuple $\left(u_{1}^{c}, u_{2}^{c}, \ldots, u_{f-1}^{c}\right)$. Since the total number of $(f-1)$-tuples of vertices of $Q$ is bounded by $n(n-1) \ldots(n-$ $(f-1)) \leqslant n^{f-1}$ we infer that there exists an $(f-1)$-tuple of vertices $\bar{u}_{1}, \bar{u}_{2}, \ldots, \bar{u}_{f-1}$ of $Q$ contained in $c n^{f} / n^{f-1} \sim c n$ copies $F^{c}$ of $F$. Consider a set $S,|S|=c n=\frac{n}{c m^{f}}$, of vertices $\bar{v}$ each of which together with $\bar{u}_{1}, \bar{u}_{2}, \ldots, \bar{u}_{f-1}$ induces a copy $F^{c}$ of $F$. Due to the $(\alpha, \delta)$-quasi-randomness of $Q$ and the fact that $\delta \leqslant \frac{\gamma}{2 m^{f}}=c \gamma, Q[S]$ is $(\alpha, \gamma)$-quasi-random and, therefore, due to the assumption of Lemma 2.2, contains a copy of $H$ with vertex set $V(H)=\left\{v_{1}, \ldots, v_{|V(H)|}\right\}$. Since each $v_{i}(1 \leqslant i \leqslant|V(H)|)$ together with $\bar{u}_{1}, \bar{u}_{2}, \ldots, \bar{u}_{f-1}$ span a copy $F^{c}$ of $F$, we infer that $\left\{\bar{u}_{1}, \bar{u}_{2}, \ldots, \bar{u}_{f-1}, v_{1}, \ldots, v_{|V(H)|}\right\}$ spans a copy of $F_{H}^{v}$. Thus, $(\alpha, \delta) \rightarrow F_{H}^{v}$

Before we prove Theorem 1.3(i) for $l=3$, we construct an auxilliary sequence of 3graphs $\mathcal{G}=\left\{G_{i}\right\}_{i=1}^{\infty}$ with density tending to $\frac{1}{4}$. We will then show that $G_{i}$ is in $Q_{n}$ for $n$ large enough. Let $G_{1}$ be a 3 -graph with three vertices and one edge. For $i>1$, let $G_{i}$ be the 3 -graph obtained by taking 3 vertex disjoint copies of $G_{i-1}$, and adding all edges with exactly one vertex in each copy. For instance, $G_{2}$ has 9 vertices and $3+3^{3}=30$ edges. 


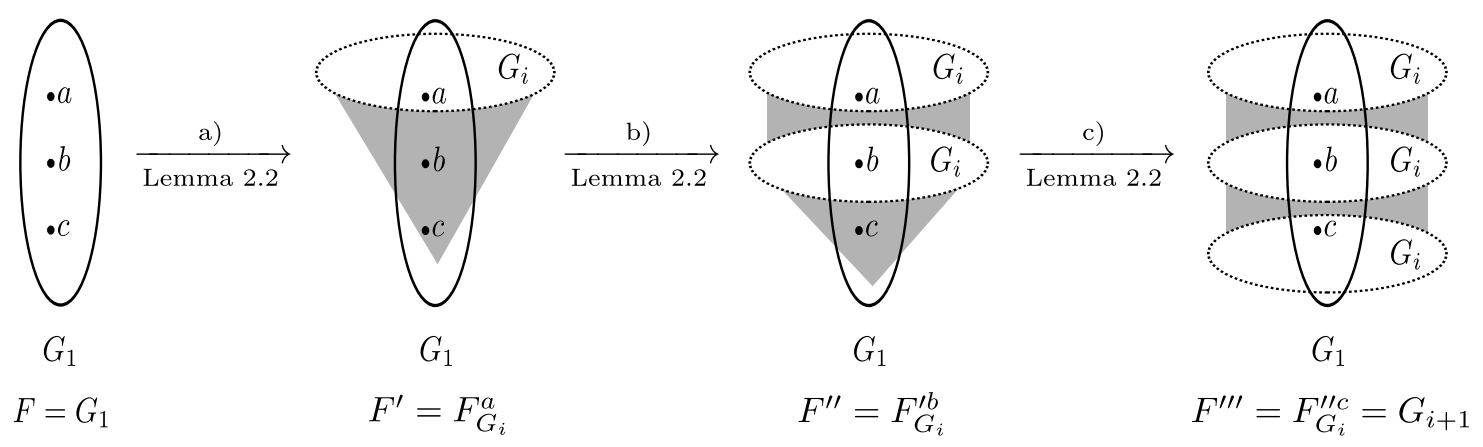

Figure 1: Three applications of Lemma 2.2 prove Claim 2.3

Since $\left|V\left(G_{i}\right)\right|=3\left|V\left(G_{i-1}\right)\right|=3^{i}$ and $\left|E\left(G_{i}\right)\right|=\left|V\left(G_{i-1}\right)\right|^{3}+3\left|E\left(G_{i-1}\right)\right|=3^{3(i-1)}\left(1+\frac{1}{9}+\right.$ $\left.\ldots \frac{1}{9^{i-1}}\right)=3^{i-1} \frac{\left(3^{i}-1\right)\left(3^{i}+1\right)}{8}$, the density of $G_{i}$ as $i \rightarrow \infty$ is

$$
\lim _{i \rightarrow \infty} d\left(G_{i}\right)=\lim _{i \rightarrow \infty} \frac{3^{i-1} \frac{\left(3^{i}-1\right)\left(3^{i}+1\right)}{8}}{\left(\begin{array}{c}
3^{i} \\
3
\end{array}\right)}=\lim _{i \rightarrow \infty} \frac{1}{4}\left(\frac{3^{i}+1}{3^{i}-2}\right)=\frac{1}{4} .
$$

Consider an arbitrary sequence of $\left(\alpha, \delta_{n}\right)$-quasi-random 3-graphs $\mathcal{Q}=\left\{Q_{n}\right\}_{n=1}^{\infty}$ with $d\left(Q_{n}\right)=\alpha\left(1 \pm \delta_{n}\right)>0$ where $\delta_{n} \in(0,1), \delta_{n}$ is decreasing and $\delta_{n} \rightarrow 0$ as $n \rightarrow \infty$. We will show that there exists $n_{1}<n_{2}<n_{3}<\ldots$ such that for $n \geqslant n_{i}, Q_{n}$ contains $G_{i}$. Based on our density calculation of $G_{i}$ above, $\bar{d}(\mathcal{Q}) \geqslant \frac{1}{4}$.

Since $Q_{n}$ contains $G_{1}$ whenever $\delta_{n}<\alpha$, it remains to show the following claim by induction on $i$ :

Claim 2.3. Assuming $\left(\alpha, \delta_{n_{i}}\right) \rightarrow G_{i}$, there exists $n_{i+1}$ such that $\left(\alpha, \delta_{n_{i+1}}\right) \rightarrow G_{i+1}$

Proof. Our goal is to find $n_{i+1}$ so that $\left(\alpha, \delta_{n}\right) \rightarrow G_{i+1}$ for all $n \geqslant n_{i+1}$. This will be achieved in three applications of Lemma 2.2 as shown in Figure 1. We will construct hypergraphs $F^{\prime}$, $F^{\prime \prime}, F^{\prime \prime \prime}$ with $G_{i} \subseteq F^{\prime} \subseteq F^{\prime \prime} \subseteq F^{\prime \prime \prime}=G_{i+1}$ and $n^{\prime}, n^{\prime \prime}, n^{\prime \prime \prime}$ with $n_{i}<n^{\prime}<n^{\prime \prime}<n^{\prime \prime \prime}=n_{i+1}$ such that

$$
\left(\alpha, \delta_{n}\right) \rightarrow F^{(i)} \text { for all } n \geqslant n^{(i)}
$$

Set $V\left(G_{1}\right)=\{a, b, c\}, H=G_{i}$, and $\gamma=\delta_{n_{i}}$. Below we will describe appropriate choices of $F, \epsilon$ and $v$ to obtain graphs $F^{(i)}, i=1,2,3$ satisfying $(*)$.

a) Set $F=G_{1}, \epsilon=\delta_{1}$ and $v=a$. Since $\left(\alpha, \delta_{1}\right) \rightarrow G_{1}$ and $\left(\alpha, \delta_{n_{i}}\right) \rightarrow G_{i}$, by Lemma 2.2 there exists $\delta^{\prime}=\delta\left(\alpha, \delta_{1}, \delta_{n_{i}}\right)$ such that $\left(\alpha, \delta^{\prime}\right) \rightarrow F_{G_{i}}^{a}$.

b) Set $F^{\prime}=F_{G_{i}}^{a}, \epsilon=\delta^{\prime}$ and $v=b$. Since $\left(\alpha, \delta^{\prime}\right) \rightarrow F^{\prime}$ and $\left(\alpha, \delta_{n_{i}}\right) \rightarrow G_{i}$, by Lemma 2.2 there exists $\delta^{\prime \prime}=\delta\left(\alpha, \delta^{\prime}, \delta_{n_{i}}\right)$ such that $\left(\alpha, \delta^{\prime \prime}\right) \rightarrow F_{G_{i}}^{\prime b}$.

c) Set $F^{\prime \prime}=F_{G_{i}}^{\prime b}, \epsilon=\delta^{\prime \prime}$ and $v=c$. Since $\left(\alpha, \delta^{\prime \prime}\right) \rightarrow F^{\prime \prime}$ and $\left(\alpha, \delta_{n_{i}}\right) \rightarrow G_{i}$, by Lemma 2.2 there exists $\delta^{\prime \prime \prime}=\delta\left(\alpha, \delta^{\prime \prime}, \delta_{n_{i}}\right)$ such that $\left(\alpha, \delta^{\prime \prime \prime}\right) \rightarrow F_{G_{i}}^{\prime \prime c}$.

Observe that $F^{\prime \prime \prime}=F_{G_{i}}^{\prime \prime \prime}=G_{i+1}$. Consequently $\left(\alpha, \delta_{n}\right) \rightarrow G_{i+1}$ for all $n$ with $\delta_{n} \leqslant \delta^{\prime \prime \prime}$. 
In a similar way to Claim 2.3 one can show a slightly more general fact stated below as Proposition 2.5. First we define the lexicographic product of two 3-graphs:

Definition 2.4. The lexicographic product of two 3-graphs $F$ and $H$ with vertex set $U$ and $W$ respectively is a 3 -graph $F \cdot H$ with vertex set $U \times W$ and with $\left\{\left(u_{1}, w_{1}\right),\left(u_{2}, w_{2}\right),\left(u_{3}, w_{3}\right)\right\} \in$ $E(F \cdot H)$ if $\left\{u_{1}, u_{2}, u_{3}\right\} \in E(F)$ or if $u_{1}=u_{2}=u_{3}$ and $\left\{w_{1}, w_{2}, w_{3}\right\} \in E(H)$.

Proposition 2.5. For all $\alpha>0, \epsilon>0, \gamma>0$ and 3-graphs $F$ and $H$ there exists $\delta=$ $\delta(\alpha, \epsilon, \gamma)>0$ such that $(\alpha, \epsilon) \rightarrow F$ and $(\alpha, \gamma) \rightarrow F$ implies $(\alpha, \delta) \rightarrow F \cdot H$.

\subsection{The upper bound for $l=3$}

It remains to show there exists a sequence of quasi-random 3-graphs with upper density $\frac{1}{4}$.

Proof. Consider a random tournament $T_{n}$ on $n$ vertices in which pairs are assigned arc direction with probability $\frac{1}{2}$. Let $R_{n}$ be a 3-graph with $V\left(R_{n}\right)=V\left(T_{n}\right)$ and $E\left(R_{n}\right)$ consisting of vertex sets of all directed 3-cycles (this 3-graph was first considered by Erdös and Hajnal in [4] in the context of Ramsey theory).

It is well known (see [3]) that $R_{n}$ is $\left(\frac{1}{4}, \delta_{n}\right)$-quasi-random with $\delta_{n} \rightarrow 0$ as $n \rightarrow \infty$. On the other hand it follows from the well known result of Kendall and Babington Smith [7] that any tournament on $n$ vertices has at most $\frac{1}{24}\left(n^{3}-n\right)$ directed 3-cycles (cf. [8]) and so no subgraph of any $R_{n}$ has density larger than $\frac{1}{4}+o(1)$. Thus the upper density of the sequence $\mathcal{R}=\left\{R_{n}\right\}_{n=1}^{\infty}$ is at most $\frac{1}{4}+o(1)$ establishing (ii) of Theorem 1.3.

\section{Proof of Theorem 1.6}

For $l=3$, Theorem $1.3(i)$ implies that every number in $\left[0, \frac{1}{4}\right)$ is a jump for quasi-random 3 -graphs. In this section, we prove that $\frac{1}{4}$ is a jump as well and, more precisely, any number in $\left[\frac{1}{4}, 0.3192\right)$ is a jump for quasi-random 3-graphs given Question 1.5 is answered positively. To this end, we use a recent result of Glebov, Král' and Volec who in [6] confirmed Question 1.5 using a computer aided proof based on Razborov's flag-algebra method [10].

Proof. Given a sequence of quasi-random 3-graphs $\mathcal{Q}=\left\{Q_{n}\right\}_{n=1}^{\infty}$ with $\bar{d}(\mathcal{Q})>\frac{1}{4}$, any $Q_{n}$ with $n \geqslant n_{0}$ contains $K_{4}^{(3)}-e$ by [6]. In a way similar to the proof of Theorem 1.3(i) we will first construct a sequence of 3 -graphs $\mathcal{F}=\left\{F_{n}\right\}_{n=1}^{\infty}$ such that $F_{n} \subseteq Q_{n}$ and $\lim _{n \rightarrow \infty} d\left(F_{n}\right)=\frac{3}{10}$. Subsequently we will alter it to a sequence of 3-graphs $\mathcal{G}=\left\{G_{n}\right\}_{n=1}^{\infty}$ in which $\lim _{n \rightarrow \infty} d\left(G_{n}\right) \approx$ 0.3192 .

Let $F_{1}=K_{4}^{(3)}-e$ with $V\left(F_{1}\right)=\left\{a_{1}, a_{2}, a_{3}, b\right\}$ and $E\left(F_{1}\right)=\left\{\left\{a_{1}, a_{2}, b\right\},\left\{a_{1}, a_{3}, b\right\}\right.$, $\left.\left\{a_{2}, a_{3}, b\right\}\right\}$. Let $A_{i}(1 \leqslant i \leqslant 3)$ and $B$ be copies of $K_{4}^{(3)}-e$. We obtain $F_{2}$ by taking four vertex disjoint copies of $F_{1}$, with vertex set $A_{i}, 1 \leqslant i \leqslant 3$, and $B$ and adding edges of type $\left\{a_{i}, a_{j}, b\right\}$ where $a_{i} \in A_{i}, a_{j} \in A_{j}, b \in B, 1 \leqslant i<j \leqslant 3$. Note that $\left|V\left(F_{2}\right)\right|=4^{2}=16$ and $\left|E\left(F_{2}\right)\right|=3(4)+4^{3}(3)$. In other words $F_{2}=F_{1} \cdot F_{1}$ is the lexicographic product of two copies of $F_{1}$. We continue in this fashion to construct the sequence $\mathcal{F}$. For $i>1$, 
let $F_{i}=F_{1} \cdot F_{i-1}$ be the 3 -graph obtained by taking four vertex disjoint copies of $F_{i-1}$, and adding edges in a similar way as described above. Since $\left|V\left(F_{i}\right)\right|=4 \mid V\left(F_{i-1} \mid=4^{i}\right.$ and $\left|E\left(F_{i}\right)\right|=3\left|V\left(F_{i-1}\right)\right|+4^{3}\left|E\left(F_{i-1}\right)\right|=3 \cdot 4^{i-1}\left(1+4^{2}+\ldots+4^{2(i-1)}\right)=\frac{4^{i-1}}{5}\left(16^{i}-1\right)$, the density of $F_{i}$ as $i \rightarrow \infty$ is

$$
\lim _{i \rightarrow \infty} d\left(F_{i}\right)=\lim _{i \rightarrow \infty} \frac{\frac{4^{i-1}}{5}\left(16^{i}-1\right)}{\left(\begin{array}{c}
4^{i} \\
3
\end{array}\right)}=\frac{3}{10} .
$$

In a similar way as in the proof of Theorem 1.3(i), one can show that for all $i$ there exists $n$ such that $F_{i}$ is contained in $Q_{n}$. Thus, every number between 0 and $\frac{3}{10}$ is a jump for quasi-random 3-graphs.

One can improve $\frac{3}{10}$ to 0.3192 by considering conveniently chosen "blow ups" of $F_{i}$. We will describe this in more detail now. Setting $V\left(F_{i}\right)=\left\{1,2, \ldots, \nu_{i}\right\}$, we first observe (similarly as in Lemma 2.2) that for each $i$, there exists an $n_{i}$ so that 3 -graphs $Q_{n}, n \geqslant n_{i}$, contain $c_{i}\left|V\left(Q_{n}\right)\right|^{\nu_{i}}$ copies of $F_{i}$. Hence by Theorem 1.1, $Q_{n}$ contains a $t$-blowup $F_{i} * t$ of $F_{i}$, more precisely, a graph with vertex set $\bigcup_{j=1}^{\nu_{i}} W_{j},\left|W_{1}\right|=\cdots=\left|W_{\nu_{i}}\right|=t$ and $\{\widetilde{a}, \widetilde{b}, \widetilde{c}\} \in$ $E\left(F_{i} * t\right)$ if $\{a, b, c\} \in E\left(F_{i}\right)$. In order to maximize the density, we consider graphs $F_{i}$ with different vertices "blown up" to sets of different cardinalities.

More precisely, set $\alpha=\frac{2}{5}(4 \sqrt{6}-9) \approx 0.2154$ and to each vertex $\bar{x}=\left(x_{1}, \ldots, x_{i}\right) \in V\left(F_{i}\right)$ assign a weight $w(\bar{x})=(1-3 \alpha)^{j} \alpha^{i-j}$ where $j$ represents the number of $b$ 's among entries of $\bar{x}$ and for $t$ large consider a blow-up $G_{i}$ of $F_{i}$ with each vertex $\bar{x}$ "blown-up" by $w(\bar{x}) * t$ vertices. Using this iterated construction, one can calculate that every number between 0 and $\frac{1}{19}(9-2 \sqrt{6}) \approx 0.3192$, where $\frac{1}{19}(9-2 \sqrt{6})=\lim _{i \rightarrow \infty} d\left(G_{i}\right)$, is a jump for quasi-random 3-graphs.

\section{Other remarks and questions}

In Section 2.2 we considered $\mathcal{R}=\left\{R_{n}\right\}_{n=1}^{\infty}$, a sequence of quasi-random 3-graphs formed by random tournaments $T_{n}$, and observed that $d(\mathcal{R})=\bar{d}(\mathcal{R})=\frac{1}{4}$. There are other quasirandom sequences of 3-graphs with density equal to upper density. Consider the quasirandom sequences $\mathcal{Q}=\left\{Q_{n}\right\}_{n=1}^{\infty}$ described in [11]: Let $\chi$ be a random $(k-1)$-coloring of pairs of $\{1, \ldots, n\}$ and define the edges of $Q_{n}$ to be all triples $\{i, u, v\}$ such that $\chi(\{i, u\}) \neq$ $\chi(\{i, v\})$. It can be shown that $d(\mathcal{Q})=\bar{d}(\mathcal{Q})=1-\frac{1}{k-1}$. In summary, if $\alpha \in\left\{\frac{1}{4}, \frac{1}{2}, \frac{2}{3}, \ldots\right\}$, then there is a sequence of quasi-random 3-graphs with $d(\mathcal{Q})=\bar{d}(\mathcal{Q})$. Are there any others?

We proved that a sequence of quasi-random l-graphs $\mathcal{Q}$ with $d(\mathcal{Q})>0$ has $\bar{d}(\mathcal{Q}) \geqslant \frac{(l-1) !}{l^{l-1}-1}$. In particular, we showed that this bound is the best possible when $l=3$. For $l=4$, it is not clear to the authors if there exists a quasi-random sequence of 4-graphs with upper density equal to $\frac{3 !}{4^{3}-1}=\frac{2}{21}$.

Theorem 1.3(i) implies that every quasi-random sequence of $l$-graphs with positive density has upper density at least $\frac{(l-1) !}{l^{l-1}-1}$. For $l=3$ this is the best possible, but we were 
unable to show an analogous fact for $l>3$. One can observe that $\frac{(l-1) !}{l^{l-1}-1}$ cannot be replaced by a number larger than $\frac{(l-1) !}{(l-1)^{l-1}}$. In order to see this, consider the quasi-random sequence $\mathcal{Q}=\left\{Q_{n}\right\}_{n=1}^{\infty}$ with vertex set $V\left(Q_{n}\right)=\{1, \ldots, n\}=[n]$. Let $\chi$ be a random $(l-1)$-coloring of pairs of $[n]$. Define the edge set $\left\{i, v_{1}, \ldots, v_{l-1}\right\} \in E\left(Q_{n}\right)$ if and only if all pairs $\left\{i, v_{1}\right\}$, $\ldots,\left\{i, v_{l-1}\right\}$ have different color. One can observe that $d(\mathcal{Q})=\bar{d}(\mathcal{Q})=\frac{(l-1) !}{(l-1)^{l-1}}$.

\section{Acknowledgements}

We would like to thank an anonymous referee for his comments and suggestions.

\section{References}

[1] N. Alon, W. de la Vega, R. Kannan, and M. Karpinski. Random sampling and approximation of MAX-CSP problems. In Proceedings of the 34th annual ACM symposium on Theory of computing, pages 232-239. ACM Press, 2002.

[2] P. Erdős. On extremal problems of graphs and generalized graphs. Israel Journal of Mathematics, 2:183-190, 1964.

[3] P. Erdős. Problems and results on graphs and hypergraphs: Similarities and differences. In J. Nešetřil and V. Rödl, editors, Mathematics of Ramsey Theory, pages 223-233. Springer-Verlag, 1990.

[4] P. Erdős and A. Hajnal. On Ramsey like theorems. Proceedings: Conference in Combinatorial Mathematics, Mathematics Institute, Oxford University, pages 123-140, 1972.

[5] P. Frankl and V. Rödl. Hypergraphs do not jump. Combinatorica, 4(2):149-159, 1984.

[6] R. Glebov, D. Král', and J. Volec. A problem of Erdős and Sós on 3-graphs. Submitted.

[7] M.G. Kendall and B. Babington Smith. On the method of paired comparisons. Biometrika, 31:324-345, 1940.

[8] J. W. Moon. Topics on Tournaments. Holt, Rhinehart and Winston, 1968.

[9] D. Mubayi and V. Rödl. Uniform edge distribution in hypergraphs is hereditary. Electronic Journal of Combinatorics, 11:\#R55, 2004.

[10] A. Razborov. Flag algebras. J. Symbolic Logic, 72(4):1239-1282, 2007.

[11] V. Rödl. On universality of graphs with uniformly distributed edges. Discrete Mathematics, 59(1-2):125-134, 1986. 